\title{
Frontiers of Inflationary Cosmology
}

\author{
Robert H. Brandenberger \\ Physics Department, Brown University, \\ Providence, R.I., USA
}

Received on 2 February, 2001

\begin{abstract}
After a brief review the theory of cosmological perturbations, I highlight some recent progress in the area of reheating in inflationary cosmology, focusing in particular on parametric amplification of super-Hubble cosmological fluctuations, and on the role of noise in the resonance dynamics (yielding a new proof of Anderson localization). I then discuss several important conceptual problems for the current realizations of inflation based on fundamental scalar matter fields, and review some new approaches at solving these problems.
\end{abstract}

\section{Introduction}

Inflationary cosmology [1] has become one of the cornerstones of modern cosmology. Inflation was the first theory within which it was possible to make predictions about the structure of the Universe on large scales based on causal physics. The development of the inflationary Universe scenario has opened up a new and extremely promising avenue for connecting fundamental physics with experiment.

After a brief introduction to inflationary cosmology (Section II) and an overview of the theory of cosmological perturbations applied to inflation (Section III), I focus on recent improvements in our understanding of the theory of inflationary reheating (Section IV), focusing in particular on recent studies of the parametric amplification of gravitational fluctuations and on the effects of noise on the resonance dynamics (which yields a new proof of Anderson localization in one spatial dimension).

However, in spite of the remarkable success of the inflationary Universe paradigm, there are several serious conceptual problems for current models in which inflation is generated by the potential energy of a scalar matter field. These problems are discussed in Section V.

Section VI is a summary of some new approaches to solving the above-mentioned problems. An attempt to obtain inflation from condensates is discussed, a nonsingular Universe construction making use of higher derivative terms in the gravitational action is explained, and a framework for calculating the back-reaction of cosmological perturbations is summarized.

This short review focuses on a selected number of topics at the forefront of inflationary cosmology. For comprehensive reviews of inflation, the reader is referred to $[2,3,4,5]$. A recent review focusing on infla- tionary model building in the context of supersymmetric models can be found in [6]. An extended and more pedagogical version of these notes is [7]. Note that Sections II, V and VI of this article are identical to the corresponding sections in [8].

\section{Basics of Inflationary Cos- mology}

Most current models of inflation are based on Einstein's theory of General Relativity with a matter source given by a scalar field $\varphi$. Based on the cosmological principle, the metric of space-time on large distance scales can be written in Friedmann-Robertson-Walker (FRW) form:

$$
d s^{2}=d t^{2}-a(t)^{2}\left[\frac{d r^{2}}{1-k r^{2}}+r^{2}\left(d \vartheta^{2}+\sin ^{2} \vartheta d \varphi^{2}\right)\right]
$$

where the constant $k$ determines the topology of the spatial sections. In the following, we shall set $k=0$, i.e. consider a spatially flat Universe. In this case, we can without loss of generality take the scale factor $a(t)$ to be equal to 1 at the present time $t_{0}$, i.e. $a\left(t_{0}\right)=1$. The coordinates $r, \vartheta$ and $\varphi$ are comoving spherical coordinates.

For a homogeneous and isotropic Universe and setting the cosmological constant to zero, the Einstein equations reduce to the FRW equations

$$
\begin{gathered}
\left(\frac{\dot{a}}{a}\right)^{2}=\frac{8 \pi G}{3} \rho \\
\frac{\ddot{a}}{a}=-\frac{4 \pi G}{3}(\rho+3 p),
\end{gathered}
$$

where $p$ and $\rho$ denote the pressure and energy density, respectively. These equations can be combined 
to yield the continuity equation (with Hubble constant $H=\dot{a} / a)$

$$
\dot{\rho}=-3 H(\rho+p) .
$$

The equation of state of matter is described by a number $w$ defined by

$$
p=w \rho .
$$

The idea of inflation [1] is very simple. We assume there is a time interval beginning at $t_{i}$ and ending at $t_{R}$ (the "reheating time") during which the Universe is exponentially expanding, i.e.,

$$
a(t) \sim e^{H t}, \quad t \epsilon\left[t_{i}, t_{R}\right]
$$

with constant Hubble expansion parameter $H$. Such a period is called "de Sitter" or "inflationary." The success of Big Bang nucleosynthesis sets an upper limit to the time $t_{R}$ of reheating, $t_{R} \ll t_{N S}, t_{N S}$ being the time of nucleosynthesis.

During the inflationary phase, the number density of any particles initially present at $t=t_{i}$ decays exponentially. At $t=t_{R}$, all of the energy which is responsible for inflation is released (see later) as thermal energy. This is a non-adiabatic process during which the entropy increases by a large factor.

A period of inflation can solve the homogeneity problem of standard cosmology, the reason being that during inflation the physical size of the forward light cone exponentially expands and thus can easily become larger than the physical size of the past light cone at $t_{r e c}$, the time of last scattering, thus explaining the near isotropy of the cosmic microwave background (CMB). Inflation also solves the flatness problem [9, 1].

Most importantly, inflation provides a mechanism which in a causal way generates the primordial perturbations required for galaxies, clusters and even larger objects. In inflationary Universe models, the Hubble radius ("apparent" horizon) and the ("actual") horizon (the forward light cone) do not coincide at late times. Provided that the duration of inflation is sufficiently long, then all scales within our present apparent horizon were inside the horizon since $t_{i}$. Thus, it is in principle possible to have a causal generation mechanism for perturbations $[10,11,12,13]$.

As will be discussed in Section III, the density perturbations produced during inflation are due to quantum fluctuations in the matter and gravitational fields $[11,12]$. The amplitude of these inhomogeneities corresponds to a temperature $T_{H} \sim H$, the Hawking temperature of the de Sitter phase. This leads one to expect that at all times $t$ during inflation, perturbations with a fixed physical wavelength $\sim H^{-1}$ will be produced. Subsequently, the length of the waves is stretched with the expansion of space, and soon becomes much larger than the Hubble radius $\ell_{H}(t)=H^{-1}(t)$. The phases of the inhomogeneities are random. Thus, the inflationary Universe scenario predicts perturbations on all scales ranging from the comoving Hubble radius at the beginning of inflation to the corresponding quantity at the time of reheating. In particular, provided that inflation lasts sufficiently long, perturbations on scales of galaxies and beyond will be generated. Note, however, that it is very dangerous to interpret de Sitter Hawking radiation as thermal radiation. In fact, the equation of state of this "radiation" is not thermal [14].

In most current models of inflation, the exponential expansion is driven by the potential energy density $V(\varphi)$ of a fundamental scalar matter field $\varphi$ with standard action

$$
\begin{aligned}
S_{m} & =\int d^{4} x \sqrt{-g} \mathcal{L}_{m} \\
\mathcal{L}_{m}(\varphi) & =\frac{1}{2} D_{\mu} \varphi D^{\mu} \varphi-V(\varphi),
\end{aligned}
$$

where $D_{\mu}$ denotes the covariant derivative, and $g$ is the determinant of the metric tensor. The resulting energymomentum tensor yields the following expressions for the energy density $\rho$ and the pressure $p$ :

$$
\begin{aligned}
& \rho(\varphi)=\frac{1}{2} \dot{\varphi}^{2}+\frac{1}{2} a^{-2}(\nabla \varphi)^{2}+V(\varphi) \\
& p(\varphi)=\frac{1}{2} \dot{\varphi}^{2}-\frac{1}{6} a^{-2}(\nabla \varphi)^{2}-V(\varphi) .
\end{aligned}
$$

It thus follows that if the scalar field is homogeneous and static, but the potential energy positive, then the equation of state $p=-\rho$ necessary for exponential inflation results (see (4)).

Most of the current realizations of potential-driven inflation are based on satisfying the conditions

$$
\dot{\varphi}^{2}, a^{-2}(\nabla \varphi)^{2} \ll V(\varphi),
$$

via the idea of slow rolling $[15,16]$. Consider the equation of motion of the scalar field $\varphi$ :

$$
\ddot{\varphi}+3 H \dot{\varphi}-a^{-2} \nabla^{2} \varphi=-V^{\prime}(\varphi) \text {. }
$$

If the scalar field starts out almost homogeneous and at rest, if the Hubble damping term (the second term on the 1.h.s. of (12) is large, and if the potential is quite flat (so that the term on the r.h.s. of (12) is small), then $\dot{\varphi}^{2}$ may remain small compared to $V(\varphi)$, in which case exponential inflation will result. Note that if the spatial gradient terms are initially negligible, they will remain negligible since they redshift.

Chaotic inflation [17] is a prototypical inflationary scenario. Consider a scalar field $\varphi$ which is very weakly coupled to itself and other fields. In this case, $\varphi$ need not be in thermal equilibrium at the Planck time, and most of the phase space for $\varphi$ will correspond to large values of $|\varphi|$ (typically $|\varphi| \gg m_{p l}$ ). Consider now a region in space where at the initial time $\varphi(x)$ is very large, and approximately homogeneous and static. In this case, the energy-momentum tensor will be immediately dominated by the large potential energy term 
and induce an equation of state $p \simeq-\rho$ which leads to inflation. Due to the large Hubble damping term in the scalar field equation of motion, $\varphi(x)$ will only roll very slowly towards $\varphi=0$ (we are making the assumption that $V(\varphi)$ has a global minimum at a finite value of $\varphi$ which can then be chosen to be $\varphi=0$ ). The kinetic energy contribution to $\rho$ and $p$ will remain small, the spatial gradient contribution will be exponentially suppressed due to the expansion of the Universe, and thus inflation persists. Note that the precise form of $V(\varphi)$ is irrelevant to the mechanism.

It is difficult to realize chaotic inflation in conventional supergravity models since gravitational corrections to the potential of scalar fields typically render the potential steep for values of $|\varphi|$ of the order of $m_{p l}$ and larger. This prevents the slow rolling condition (11) from being realizable. Even if this condition can be satisfied, there are constraints from the amplitude of produced density fluctuations which are much harder to satisfy (see Section V).

Hybrid inflation [18] is a solution to the abovementioned problem of chaotic inflation. Hybrid inflation requires at least two scalar fields to play an important role in the dynamics of the Universe. As a toy model, consider the potential of a theory with two scalar fields $\varphi$ and $\psi$ :

$$
V(\varphi, \psi)=\frac{1}{4} \lambda\left(M^{2}-\psi^{2}\right)^{2}+\frac{1}{2} m^{2} \varphi^{2}+\frac{1}{2} \lambda^{\prime} \psi^{2} \varphi^{2}
$$

For values of $|\varphi|$ larger than $\varphi_{c}$

$$
\varphi_{c}=\left(\frac{\lambda}{\lambda^{\prime}} M^{2}\right)^{1 / 2}
$$

the minimum of $\psi$ is $\psi=0$, whereas for smaller values of $\varphi$ the symmetry $\psi \rightarrow-\psi$ is broken and the ground state value of $|\psi|$ tends to $M$. The idea of hybrid inflation is that $\varphi$ is slowly rolling like the inflaton field in chaotic inflation, but that the energy density of the Universe is dominated by $\psi$. Inflation terminates once $|\varphi|$ drops below the critical value $\varphi_{c}$, at which point $\psi$ starts to move.

Note that in hybrid inflation $\varphi_{c}$ can be much smaller than $m_{p l}$ and hence inflation without super-Planck scale values of the fields is possible. It is possible to implement hybrid inflation in the context of supergravity (see e.g. [19]).

At the present time there are many realizations of potential-driven inflation, but there is no canonical theory. A lot of attention is being devoted to implementing inflation in the context of unified theories, the prime candidate being superstring theory or M-theory. String theory or M-theory live in 10 or 11 space-time dimensions, respectively. When compactified to 4 space-time dimensions, there exist many moduli fields, scalar fields which describe flat directions in the complicated vacuum manifold of the theory. A lot of attention is now devoted to attempts at implementing inflation using moduli fields (see e.g. [20] and references therein).

Recently, it has been suggested that our space-time is a brane in a higher-dimensional space-time (see [21] for the basic construction). Ways of obtaining inflation on the brane are also under active investigation (see e.g. [22]).

It should also not be forgotten that inflation can arise from the purely gravitational sector of the theory, as in the original model of Starobinsky [23] (see also Section VI), or that it may arise from kinetic terms in an effective action as in pre-big-bang cosmology [24] or in k-inflation [25].

Theories with (almost) exponential inflation generically predict an (almost) scale-invariant spectrum of density fluctuations, as was first realized in $[10,11,12$, $13]$ and then studied more quantitatively in [26, 27, 28]. Via the Sachs-Wolfe effect [29], these density perturbations induce $\mathrm{CMB}$ anisotropies with a spectrum which is also scale-invariant on large angular scales.

The heuristic picture is as follows. If the inflationary period which lasts from $t_{i}$ to $t_{R}$ is almost exponential, then the physical effects which are independent of the small deviations from exponential expansion (an example of something which does depend on these deviations is effects connected with the remnant radiation density during inflation) are time-translation-invariant. This implies, for example, that quantum fluctuations at all times have the same strength when measured on the same physical length scale.

If the inhomogeneities are small, they can described by linear theory, which implies that all Fourier modes $k$ evolve independently. The exponential expansion inflates the wavelength of any perturbation. Thus, the wavelength of perturbations generated early in the inflationary phase on length scales smaller than the Hubble radius soon becomes equal to the ("exits") Hubble radius (this happens at the time $t_{i}(k)$ ) and continues to increase exponentially. After inflation, the Hubble radius increases as $t$ while the physical wavelength of a fluctuation increases only as $a(t)$. Thus, eventually the wavelength will cross the Hubble radius again (it will "enter" the Hubble radius) at time $t_{f}(k)$. Thus, it is possible for inflation to generate fluctuations on cosmological scales by causal physics.

Any physical process which obeys the symmetry of the inflationary phase and which generates perturbations will generate fluctuations of equal strength when measured when they cross the Hubble radius (see, however, Section V.2):

$$
\frac{\delta M}{M}\left(k, t_{i}(k)\right)=\mathrm{const}
$$

(independent of $k$ ). Here, $\delta M(k, t)$ denotes the r.m.s. mass fluctuation on a length scale $k^{-1}$ at time $t$.

It is generally assumed that causal physics cannot affect the amplitude of fluctuations on super-Hubble 
scales (see, however, the comments at the end of Section IV.1). Therefore, the magnitude of $\frac{\delta M}{M}$ can change only by a factor independent of $k$, and hence it follows that

$$
\frac{\delta M}{M}\left(k, t_{f}(k)\right)=\text { const },
$$

which is the definition of a scale-invariant spectrum [30].

\section{Theory of Cosmological Per- turbations}

On scales larger than the Hubble radius the Newtonian theory of cosmological perturbations is inapplicable, and a general relativistic analysis is needed. On these scales, matter is essentially frozen in comoving coordinates. However, space-time fluctuations can still increase in amplitude.

In principle, it is straightforward to work out the general relativistic theory of linear fluctuations [31]. We linearize the Einstein equations

$$
G_{\mu \nu}=8 \pi G T_{\mu \nu}
$$

(where $G_{\mu \nu}$ is the Einstein tensor associated with the space-time metric $g_{\mu \nu}$, and $T_{\mu \nu}$ is the energymomentum tensor of matter) about an expanding FRW background $\left(g_{\mu \nu}^{(0)}, \varphi^{(0)}\right)$ :

$$
\begin{aligned}
g_{\mu \nu}(\underline{x}, t) & =g_{\mu \nu}^{(0)}(t)+h_{\mu \nu}(\underline{x}, t) \\
\varphi(\underline{x}, t) & =\varphi^{(0)}(t)+\delta \varphi(\underline{x}, t)
\end{aligned}
$$

and pick out the terms linear in $h_{\mu \nu}$ and $\delta \varphi$ to obtain

$$
\delta G_{\mu \nu}=8 \pi G \delta T_{\mu \nu}
$$

In the above, $h_{\mu \nu}$ is the perturbation in the metric and $\delta \varphi$ is the fluctuation of the matter field $\varphi$.

In practice, there are many complications which make this analysis highly nontrivial. The first problem is "gauge invariance" [32]. Imagine starting with a homogeneous FRW cosmology and introducing new coordinates which mix $\underline{x}$ and $t$. In terms of the new coordinates, the metric now looks inhomogeneous. The inhomogeneous piece of the metric, however, must be a pure coordinate (or "gauge") artifact. Thus, when analyzing relativistic perturbations, care must be taken to factor out effects due to coordinate transformations.

There are various methods of dealing with gauge artifacts. The simplest and most physical approach is to focus on gauge invariant variables, i.e., combinations of the metric and matter perturbations which are invariant under linear coordinate transformations.

The gauge invariant theory of cosmological perturbations is in principle straightforward, although technically rather tedious. In the following I will summarize the main steps and refer the reader to [33] for the details and further references (see also [34] for a pedagogical introduction and [35, 36, 37, 38, 39, 40, 41, 42] for other approaches).

We consider perturbations about a spatially flat Friedmann-Robertson-Walker metric

$$
d s^{2}=a^{2}(\eta)\left(d \eta^{2}-d \underline{x}^{2}\right)
$$

where $\eta$ is conformal time (related to cosmic time $t$ by $a(\eta) d \eta=d t)$. At the linear level, metric perturbations can be decomposed into scalar modes, vector modes and tensor modes (gravitational waves). In the following, we will focus on the scalar modes since they are the only ones which couple to energy density and pressure. A scalar metric perturbation (see [43] for a precise definition) can be written in terms of four free functions of space and time:

$$
\delta g_{\mu \nu}=a^{2}(\eta)\left(\begin{array}{cc}
2 \phi & -B_{, i} \\
-B_{, i} & 2\left(\psi \delta_{i j}+E_{, i j}\right)
\end{array}\right) .
$$

The next step is to consider infinitesimal coordinate transformations which preserve the scalar nature of $\delta g_{\mu \nu}$, and to calculate the induced transformations of $\phi, \psi, B$ and $E$. Then we find invariant combinations to linear order. (Note that there are in general no combinations which are invariant to all orders [44].) After some algebra, it follows that

$$
\begin{aligned}
& \Phi=\phi+a^{-1}\left[\left(B-E^{\prime}\right) a\right]^{\prime} \\
& \Psi=\psi-\frac{a^{\prime}}{a}\left(B-E^{\prime}\right)
\end{aligned}
$$

are two invariant combinations (a prime denotes differentiation with respect to $\eta$ ).

Perhaps the simplest way [33] to derive the equations of motion for gauge invariant variables is to consider the linearized Einstein equations (20) and to write them out in the longitudinal gauge defined by $B=E=0$, in which $\Phi=\phi$ and $\Psi=\psi$, to directly obtain gauge invariant equations.

For several types of matter, in particular for scalar field matter, $\delta T_{j}^{i} \sim \delta_{j}^{i}$ which implies $\Phi=\Psi$. Hence, the scalar-type cosmological perturbations can in this case be described by a single gauge invariant variable. In the case of a single scalar matter field $\varphi$, the perturbed Einstein equations can be combined to yield

$$
\ddot{\Phi}+\left(H-2 \frac{\ddot{\varphi}}{\dot{\varphi}}\right) \dot{\Phi}+\left(\frac{k^{2}}{a^{2}}+2 \dot{H}-2 H \frac{\ddot{\varphi}}{\dot{\varphi}}\right) \Phi=0 .
$$

For fluctuations with scales larger than the Hubble radius, this equation of motion can be written in the form of an approximate conservation law [28, 45, 39, 46, 47, 48]

$$
\dot{\varphi}^{2} \dot{\xi}=0
$$

where

$$
\xi=\frac{2}{3} \frac{H^{-1} \dot{\Phi}+\Phi}{1+w}+\Phi .
$$


During the period of slow-rolling of the scalar field (and also for single perfect fluids), (26) becomes simply $\dot{\xi}=0$.

If the equation of state of matter is constant, i.e., $w=$ const, then $\dot{\xi}=0$ implies that the relativistic potential is time-independent on scales larger than the Hubble radius, i.e. $\Phi(t)=$ const. During a transition from an initial phase with $w=w_{i}$ to a phase with $w=w_{f}, \Phi$ changes. In many cases, a good approximation to the dynamics given by $(26)$ is

$$
\frac{\Phi}{1+w}\left(t_{i}\right)=\frac{\Phi}{1+w}\left(t_{f}\right)
$$

To make contact with late time matter perturbations and with the Newtonian intuition, it is useful to note that, as a consequence of the Einstein constraint equations, at the time $t_{H}(k)$ when a mode $k$ crosses the Hubble radius, $\Phi$ is a measure of the fractional density fluctuations:

$$
\Phi\left(k, t_{H}(k)\right) \sim \frac{\delta \rho}{\rho}\left(k, t_{H}(k)\right) .
$$

The primordial perturbations in an inflationary cosmology are generated by quantum fluctuations (see also $[49,50])$. Since the scale of the fluctuations of interest today was larger than the Hubble radius for a long time, it is crucial to consider not just matter fluctuations, but also the gravitational fluctuations described at a classical level in the previous paragraphs. Thus, the generation and evolution of cosmological fluctuations in inflationary cosmology becomes a problem of quantum gravity. However, due to the fact that gravity is an attractive force, we know that the amplitude of the fluctuations had to have been extremely small in the very early Universe. Hence, a perturbative analysis will be well justified. What follows is a very brief summary of the unified analysis of the quantum generation and evolution of perturbations in an inflationary Universe (for a detailed review see [33]). The basic point is that at the linearized level, the system of gravitational and matter perturbations can be quantized in a consistent way. The use of gauge invariant variables makes the analysis both physically clear and computationally simple. Due to the Einstein constraint equation which couples metric and matter fluctuations, there is only one scalar field degree of freedom to be quantized (see [51] and [52] for the original analysis).

The first step of this analysis is to expand the gravitational and matter actions to quadratic order in the fluctuation variables about a classical homogeneous and isotropic background cosmology. Focusing on the scalar metric sector, it turns out that one can express the resulting action for the quantum fluctuations in terms of a single gauge invariant variable which is a combination of metric and matter perturbations, and that the resulting action reduces to the action of a single gauge invariant free scalar field with a time dependent mass
$[52,51]$ (the time dependence reflects the expansion of the background space-time) We can thus use standard methods to quantize this theory. If we employ canonical quantization, then the mode functions of the field operator obey the same equations as we derived in the gauge-invariant analysis of classical relativistic perturbations.

The time dependence of the mass leads to equations which have growing modes which correspond to particle production or equivalently to the generation and amplification of fluctuations. Since inflation exponentially dilutes the density of pre-existing matter, it is reasonable to assume that the perturbations start off (e.g. at the beginning of inflation) in the vacuum state (defined as a state with no particles with respect to a local comoving observer). The state defined this way will not be the vacuum state from the point of view of an observer at a later time. The Bogoliubov mode mixing technique can be used to calculate the number density of particles at a later time. In particular, expectation values of field operators such as the power spectrum can be computed.

If the background scalar field is rolling slowly, then the resulting mass fluctuations are given by

$$
\frac{\delta M}{M}\left(k, t_{f}(k)\right) \sim \frac{3 H^{2}\left|\dot{\varphi}_{0}\left(t_{i}(k)\right)\right|}{\dot{\varphi}_{0}^{2}\left(t_{i}(k)\right)}=\frac{3 H^{2}}{\left|\dot{\varphi}_{0}\left(t_{i}(k)\right)\right|}
$$

This result can now be evaluated for specific models of inflation to find the conditions on the particle physics parameters which give a value

$$
\frac{\delta M}{M}\left(k, t_{f}(k)\right) \sim 10^{-5}
$$

which is required if quantum fluctuations from inflation are to provide the seeds for galaxy formation and agree with the CMB anisotropy data.

For chaotic inflation with a potential

$$
V(\varphi)=\frac{1}{2} m^{2} \varphi^{2}
$$

we can solve the slow rolling equations for the inflaton and obtain the requirement $m \sim 10^{13} \mathrm{GeV}$ to agree with (31). Similarly, for a quartic potential with coupling constant $\lambda$, the condition $\lambda \leq 10^{-12}$ is required in order not to conflict with observations. Thus, in both examples one needs a very small parameter in the particle physics model. It has been shown quite generally [53] that small parameters are required if inflation is to solve the fluctuation problem.

To summarize, the main results of the analysis of density fluctuations in inflationary cosmology are: (1) Quantum vacuum fluctuations in the de Sitter phase of an inflationary Universe are the source of perturbations. (2) As a consequence of the change in the background equation of state, the evolution outside the Hubble radius produces a large amplification of the perturbations. 
In fact, unless the particle physics model contains very small coupling constants, the predicted fluctuations are in excess of those allowed by the bounds on cosmic microwave anisotropies. (3) The quantum generation and classical evolution of fluctuations can be treated in a unified manner. The formalism is no more complicated that the study of a free scalar field in a time dependent background. (4) Inflationary Universe models generically produce an approximately scale invariant Harrison-Zel'dovich spectrum (16).

\section{Parametric Resonance and Reheating}

Reheating is an important stage in inflationary cosmology. It determines the state of the Universe after inflation and has consequences for baryogenesis, defect formation and other aspects of cosmology.

After slow rolling, the inflaton field begins to oscillate uniformly in space about the true vacuum state. Quantum mechanically, this corresponds to a coherent state of $k=0$ inflaton particles. Due to interactions of the inflaton with itself and with other fields, the coherent state will decay into quanta of elementary particles. This corresponds to post-inflationary particle production.

Reheating is usually studied using simple scalar field toy models. The one we will adopt here consists of two real scalar fields, the inflaton $\varphi$ interacting with a massless scalar field $\chi$ representing ordinary matter. The Lagrangian is

$$
\mathcal{L}=\frac{1}{2} \partial_{\mu} \varphi \partial^{\mu} \varphi-\frac{1}{2} m^{2} \varphi^{2}+\frac{1}{2} \partial_{\mu} \chi \partial^{\mu} \chi-\frac{1}{2} g^{2} \varphi^{2} \chi^{2}
$$

with $m \sim 10^{13} \mathrm{GeV}$ (see Section III for a justification of this choice), and $g^{2}$ denoting the interaction coupling constant. The bare mass and self interactions of $\chi$ are neglected.

In the elementary theory of reheating (see e.g. [54] and [55]), the decay of the inflaton was calculated using first order perturbation theory. The decay rate $\Gamma_{B}$ of $\varphi$ typically turns out to be much smaller than the Hubble expansion rate at the end of inflation (see [7] for a worked example). The decay leads to a decrease in the amplitude of $\varphi$ which can be approximated by adding an extra damping term to the equation of motion for $\varphi:$

$$
\ddot{\varphi}+3 H \dot{\varphi}+\Gamma_{B} \dot{\varphi}=-V^{\prime}(\varphi) .
$$

From the above equation it follows that as long as $H>\Gamma_{B}$, particle production is negligible. During the phase of coherent oscillation of $\varphi$, the energy density and hence $H$ are decreasing. Thus, eventually $H=\Gamma_{B}$, and at that point reheating occurs (the remaining energy density in $\varphi$ is very quickly transferred to $\chi$ particles). However, when this occurs, the matter temperature is much smaller than the energy scale of inflation (reheating is a slow process). This would imply no GUT baryogenesis and no GUT-scale defect production. As we shall see, these conclusions change radically if we adopt an improved analysis of reheating.

As was first realized in [56], the above analysis misses an essential point. To see this, we focus on the equation of motion for the matter field $\chi$. The equations for the Fourier modes $\chi_{k}$ in the presence of a coherent inflaton field oscillating with amplitude $A$,

$$
\varphi(t)=A \cos (m t)
$$

is

$$
\ddot{\chi}_{k}+3 H \dot{\chi}_{k}+\left(k_{p}^{2}+m_{\chi}^{2}+\frac{1}{2} g^{2} A^{2} \cos (2 m t)\right) \chi_{k}=0,
$$

where $k_{p}=k / a$ is the time-dependent physical wavenumber, and $m_{\chi}^{2}=\frac{1}{2} A^{2}$ (for other toy models a similar equation is obtained, but with a different relationship between the mass and the coefficient of the oscillating term).

Let us for the moment neglect the expansion of the Universe. In this case, the friction term in (36) drops out, $k_{p}$ is time-independent, and Equation (36) becomes a harmonic oscillator equation with a periodically varying mass. In the mathematics literature, this equation is called the Mathieu equation. It is well known that there is an instability. In physics, the effect is known as parametric resonance (see e.g. [57]). At frequencies $\omega_{n}$ corresponding to half integer multiples of the frequency $\omega$ of the variation of the mass, i.e.

$$
\omega_{k}^{2}=k_{p}^{2}+m_{\chi}^{2}=\left(\frac{n}{2} \omega\right)^{2} \quad n=1,2, \ldots,
$$

there are instability bands with widths $\Delta \omega_{n}$. For values of $\omega_{k}$ within the instability band, the value of $\chi_{k}$ increases exponentially:

$$
\chi_{k} \sim e^{\mu t} \text { with } \mu \sim \frac{g^{2} A^{2}}{\omega} .
$$

In models of chaotic inflation $A \sim m_{p l}$. Hence, unless $g^{2}$ is unnaturally small (a typical value is $g^{2} \sim m / m_{p l}$ ), it follows that $\mu \gg H$. The constant $\mu$ is called the Floquet exponent.

Since the widths of the instability bands decrease as a power of the (small) coupling constant $g^{2}$ with increasing $n$, for practical purposes only the lowest instability band is important. Its width is

$$
\Delta \omega_{k} \sim g A
$$

Note, in particular, that there is no ultraviolet divergence in computing the total energy transfer from the $\varphi$ to the $\chi$ field due to parametric resonance [56].

It is easy to include the effects of the expansion of the Universe (see e.g. [56, 58, 59]). The main effect is that the value of $\omega_{k}$ becomes time-dependent. Thus, a 
mode slowly enters and leaves the resonance bands. As a consequence, any mode lies in the resonance band for only a finite time.

The rate of energy transfer is given by the phase space volume of the lowest instability band multiplied by the rate of growth of the mode function $\chi_{k}$. Using as an initial condition for $\chi_{k}$ the value $\chi_{k} \sim H$ given by the magnitude of the expected quantum fluctuations, we obtain

$$
\dot{\rho} \sim \mu\left(\frac{\omega}{2}\right)^{2} \Delta \omega_{k} H e^{\mu t} .
$$

Hence, the energy transfer will proceed fast on the time scale of the expansion of the Universe. There will be explosive particle production, and the energy density in matter at the end of reheating will be approximately equal to the energy density at the end of inflation.

The above is a summary of the main physics of the modern theory of reheating. The actual analysis can be refined in many ways (see e.g. $[58,59,60]$, and, in the toy model considered here, [61]). First of all, it is easy to take the expansion of the Universe into account explicitly (by means of a transformation of variables), to employ an exact solution of the background model and to reduce the mode equation for $\chi_{k}$ to an equation which also admits exponential instabilities.

The next improvement consists of treating the $\chi$ field quantum mechanically (keeping $\varphi$ as a classical background field). At this point, the techniques of quantum field theory in a curved background can be applied. There is no need to impose artificial classical initial conditions for $\chi_{k}$. Instead, we may assume that $\chi$ starts in its initial vacuum state. The Bogoliubov mode mixing technique can be used to compute the number of particles at late times.

Note that the state of $\chi$ after parametric resonance is not a thermal state. The spectrum consists of high peaks in distinct wave bands. An important question is how this state thermalizes. For some recent progress on this issue see $[62,63]$. Since the state after explosive particle production is not a thermal state, it is useful to follow [58] and call this process "preheating" instead of reheating.

Note that the details of the analysis of preheating are quite model-dependent. In fact [58, 60], in most models one does not get the kind of "narrow-band" resonance discussed here, but "broad-band" resonance. In this case, the energy transfer is even more efficient.

Recently [64] it has been argued that parametric resonance may lead to resonant amplification of superHubble-scale cosmological perturbations. The point is that in the presence of an oscillating inflaton field, the equation of motion (25) for the cosmological perturbations contains a contribution to the mass term (the coefficient of $\Phi)$ which is periodically varying in time. Hence, the equation takes on a similar form to the Mathieu equation discussed above (36). In some models of inflation, the first resonance band includes modes with wavelength larger than the Hubble radius, leading to the apparent amplification of super-Hubble-scale modes. Such a process does not violate causality [48] since it is driven by the inflaton field which is coherent on super-Hubble scales at the end of inflation as a consequence of the causal dynamics of an inflationary Universe.

The analysis of Equation (25) during the period of reheating is, however, complicated by a singularity in the coefficients of both $\dot{\Phi}$ and $\Phi$ at the turning points of the scalar matter field $\varphi$. This singularity persists when using the 'conservation law' form (26) of the equation: when $\dot{\varphi}=0$, one cannot immediately draw the conclusion that $\dot{\chi}=0$. Note, also, that a large increase in the value of $\Phi$ during reheating is predicted by the usual theory of cosmological fluctuations which treats the reheating period as a smooth change in the equation of state from that of nearly de Sitter to that of a radiation-dominated Universe (see (28)). Careful analyses for simple single-field [48, 65] models demonstrated that there is indeed no net growth of the physical amplitude of gravitational fluctuations beyond what the usual theory of cosmological perturbations predicts (see also $[66,67]$ for earlier results supporting this conclusion). There is increasing evidence that this conclusion holds in general for models with purely adiabatic perturbations [68, 69].

In the case of multiple matter field models there are extra terms on the right hand side of the equations of motion (25) and (26) which are not exponentially suppressed on length scales larger than the Hubble radius. These terms are related to the existence of isocurvature fluctuations. As first demonstrated in [73], in such models exponential increase in the amplitude of $\chi$ during reheating is indeed possible. However, in many models the perturbation modes which can undergo parametric amplification during reheating are exponentially suppressed during inflation [70, 71, 72], and they thus have a negligible effect on the final amplitude of $\chi$. The criterion for models (such as the one proposed in [73]) to have exponential growth of the physical amplitude of cosmological perturbations during inflation is that there is an isocurvature/entropy mode which is not suppressed during inflation [74]. The resulting exponential amplification of fluctuations renders these models incompatible with the observational constraints, even including back-reaction effects [75].

Since the exponential particle production rate during reheating relies on a parametric resonance instability, it is reasonable to be concerned whether the effect will survive in the presence of noise. There are various sources of noise to be concerned about. Firstly, there are the quantum or thermal fluctuations in the inflaton itself, the fluctuations which in inflationary cosmology are the source of the observed structure in the Universe. There is also noise and associated dissipation due to the coupling of the $\chi$ field to other fields. In $[76,77]$ we have 
considered the effects of noise in the inflaton field on the dynamics of $\chi$.

We assume that the dynamics of the inflaton is described by periodic motion with superimposed noise given by a function $q(t, x)$, i.e.

$$
\varphi(t, x)=A \cos (\omega t)+q(t, x) .
$$

For simplicity, we have neglected the expansion of the Universe. In the case of spatially homogeneous noise considered in [76], the dynamics of $\chi$ still decomposes into independent Fourier modes which obey the equation

$$
\ddot{\chi}_{k}+k^{2} \chi_{k}+\left[m_{\chi}^{2}+p(\omega t)+q(t)\right] \chi_{k}=0,
$$

where $p(y)$ is a function with period $2 \pi$. This equation can be written in the usual way as a homogeneous first order $2 \times 2$ matrix differential equation with a coefficient matrix containing noise.

For deriving the results mentioned below, it is sufficient to make certain statistical assumptions about $q(t)$. We assume that the noise is drawn from some sample space $\Omega$ (which for homogeneous noise can be taken to be $\Omega=C(\Re)$ ), and that the noise is ergodic, i.e. the time average of the noise is equal to the expectation value of the noise over the sample space. In this case, it can be shown that the generalized Floquet exponent of the solutions for $\chi_{k}$ is well defined. To obtain more quantitative information about $\mu(q)$ it is necessary to make further assumptions about the noise. We assume firstly that the noise $q(t)$ is uncorrelated in time on scales larger than the time period $T$ of the periodic motion given by $p$, and is identically distributed for all realizations of the noise. Secondly, we assume that restricting the noise $q(t ; \kappa)$ to the time interval $0 \leq t<T$, the noise samples within the support of the probability measure fill a neighborhood, in $C(0, T)$, of the origin.

The main result which was proved in [76] is that the Floquet exponent $\mu(q)$ in the presence of noise is strictly larger than the exponent $\mu(0)$ in the absence of noise

$$
\mu(q)>\mu(0)
$$

which demonstrates that the presence of noise leads to a strict increase in the rate of particle production. The proof was based on an application of Furstenberg's theorem, a theorem concerning the Lyapunov exponent of products of independent identically distributed random matrices $\left\{\Psi_{j}: j=1, \ldots, N\right\}$.

For inflationary reheating, the above result implies that noise in the inflaton eliminated the stability bands of the system, and that all modes $\chi_{k}$ grow exponentially. The result was extended to inhomogeneous noise in [77]. The analysis is mathematically much more complicated since the Fourier modes no longer decouple and the problem is a problem in the theory of partial (rather than ordinary) differential equations. Nevertheless, a similar result to (43) can be derived, with the strict inequality replaced by $\geq$.

If we replace time derivatives by spatial derivatives (denoted by a prime) in (42), we obtain the time-independent Schrödinger equation for a onedimensional non-relativistic electron gas in a periodic potential subject to aperiodic noise

$$
-\tilde{E} \psi(x)=-\psi^{\prime \prime}+\left[V_{p}(x)+V_{q}(x)\right] \psi,
$$

where $V_{p}$ is periodic, $V_{q}$ represents the random noise contribution to the potential, and $\tilde{E}$ is a constant. Our results imply that in the presence of random noise, the periodic solutions of the equation with periodic potential (the Bloch waves) become localized, and that the localization length decreases as the noise amplitude increases [78]. Thus, we obtain a new proof of Anderson localization [79].

\section{Problems of Inflationary Cos- mology}

\section{V.A Fluctuation Problem}

A generic problem for all realizations of potentialdriven inflation studied up to now concerns the amplitude of the density perturbations which are induced by quantum fluctuations during the period of exponential expansion [27, 28]. From the amplitude of CMB anisotropies measured by COBE, and from the present amplitude of density inhomogeneities on scales of clusters of galaxies, it follows that the amplitude of the mass fluctuations $\delta M / M$ on a length scale given by the comoving wavenumber $k$ at the time $t_{f}(k)$ when that scale crosses the Hubble radius in the FRW period is of the order $10^{-5}$.

However, as was discussed in detail in Section III, the present realizations of inflation based on scalar quantum field matter generically [53] predict a much larger value of these fluctuations, unless a parameter in the scalar field potential takes on a very small value. For example, as discussed at the end of Section III, in a single field chaotic inflationary model with quartic potential the mass fluctuations generated are of the order $10^{2} \lambda^{1 / 2}$. Thus, in order not to conflict with observations, a value of $\lambda$ smaller than $10^{-12}$ is required. There have been many attempts to justify such small parameters based on specific particle physics models, but no single convincing model has emerged.

With the recent discovery $[64,48]$ that long wavelength gravitational fluctuations may be amplified exponentially during reheating, a new aspect of the fluctuation problem has emerged. All models in which such amplification occurs (see e.g. [74] for a discussion of the required criteria) are ruled out because the amplitude of the fluctuations after back-reaction has set in is too 
large, independent of the value of the coupling constant $[75]$.

\section{V.B Trans-Planckian Problem}

In many models of inflation, in particular in chaotic inflation, the period of inflation is so long that comoving scales of cosmological interest today corresponded to a physical wavelength much smaller than the Planck length at the beginning of inflation. In extrapolating the evolution of cosmological perturbations according to linear theory to these very early times, we are implicitly making the assumptions that the theory remains perturbative to arbitrarily high energies and that it can be described by classical general relativity. Both of these assumptions break down on super-Planck scales. Thus the question arises as to whether the predictions of the theory are robust against modifications of the model on super-Planck scales.

A similar problem occurs in black hole physics [80]. The mixing between the modes falling towards the black hole from past infinity and the Hawking radiation modes emanating to future infinity takes place in the extreme ultraviolet region, and could be sensitive to super-Planck physics. However, in the case of black holes it has been shown that for sub-Planck wavelengths at future infinity, the predictions do not change under a class of drastic modifications of the physics described by changes in the dispersion relation of a free field [81, 82].

As was recently $[83,84]$ discovered, the result in the case of inflationary cosmology is different: the spectrum of fluctuations may depend quite sensitively on the dispersion relation on super-Planck scales. If we take the initial state of the fluctuations at the beginning of inflation to be given by the state which minimizes the energy density, then for certain of the dispersion relations considered in [82], the spectrum of fluctuations changes quite radically. The index of the spectrum can change (i.e. the spectrum is no longer scale-invariant) and oscillations in the spectrum may be induced. Note that for the class of dispersion relations considered in [81] the predictions are the standard ones.

The above results may be bad news for people who would like to consider scalar-field driven inflationary models as the ultimate theory. However, the positive interpretation of the results is that the spectrum of fluctuations may provide a window on super-Planck-scale physics. The present observations can already be interpreted in the sense [85] that the dispersion relation of the effective field theory which emerges from string theory cannot differ too drastically from the dispersion relation of a free scalar field.

\section{V.C Singularity Problem}

Scalar field-driven inflation does not eliminate singularities from cosmology. Although the standard assumptions of the Penrose-Hawking theorems break down if matter has an equation of state with negative pressure, as is the case during inflation, nevertheless it can be shown that an initial singularity persists in inflationary cosmology [86]. This implies that the theory is incomplete. In particular, the physical initial value problem is not defined.

\section{V.D Cosmological Constant Problem}

Since the cosmological constant acts as an effective energy density, its value is bounded from above by the present energy density of the Universe. In Planck units, the constraint on the effective cosmological constant $\Lambda_{\text {eff }}$ is (see e.g. [87])

$$
\frac{\Lambda_{e f f}}{m_{p l}^{4}} \leq 10^{-122} .
$$

This constraint applies both to the bare cosmological constant and to any matter contribution which acts as an effective cosmological constant.

The true vacuum value (taken on, to be specific, at $\varphi=0$ ) of the potential $V(\varphi)$ acts as an effective cosmological constant. Its value is not constrained by any particle physics requirements (in the absence of special symmetries). Thus there must be some as yet unknown mechanism which cancels (or at least almost completely cancels) the gravitational effects of any vacuum potential energy of any scalar field. However, scalar fielddriven inflation relies on the almost constant potential energy $V(\varphi)$ during the slow-rolling period acting gravitationally. How can one be sure that the unknown mechanism which cancels the gravitational effects of $V(0)$ does not also cancel the gravitational effects of $V(\varphi)$ during the slow-rolling phase? This problem is the Achilles heel of any scalar field-driven inflationary model.

Supersymmetry alone cannot provide a resolution of this problem. It is true that unbroken supersymmetry forces $V(\varphi)=0$ in the supersymmetric vacuum. However, supersymmetry breaking will induce a nonvanishing $V(\varphi)$ in the true vacuum after supersymmetry breaking, and a cosmological constant problem of at least 60 orders of magnitude remains even with the lowest scale of supersymmetry breaking compatible with experiments.

\section{New Avenues}

In the light of the problems of potential-driven inflation discussed in the previous sections, it is of utmost importance to study realizations of inflation which do not require fundamental scalar fields, or completely new avenues towards early Universe cosmology which, while maintaining (some of) the successes of inflation, address and resolve some of its difficulties.

Two examples of new avenues to early Universe cosmology which do not involve conventional inflation are 
the pre-big-bang scenario [24], and the varying speed of light postulate $[88,89]$. The pre-big-bang scenario is a model in which the Universe starts in an empty and flat dilaton-dominated phase which leads to pole-law inflation. A nice feature of this theory is that the mechanism of super-inflationary expansion is completely independent of a potential and thus independent of the cosmological constant issue. The scenario, however, is confronted with a graceful exit problem [90], and the initial conditions need to be very special [91] (see, however, the discussion in [92]).

It is also easy to realize that a theory in which the speed of light is much larger in the early Universe than at the present time can lead to a solution of the horizon and flatness problems of standard cosmology and thus can provide an alternative to inflation for addressing them. For realizations of this scenario in the context of the brane world ideas see e.g. [93, 94, 95].

String theory may lead to a natural resolution of some of the puzzles of inflationary cosmology. This is an area of active research. The reader is referred to [20] for a review of recent studies of obtaining inflation with moduli fields, and to [22] for attempts to obtain inflation with branes. Below, three more conventional approaches to addressing some of the problems of inflation will be summarized.

\section{VI.A Inflation from Condensates}

At the present time there is no direct observational evidence for the existence of fundamental scalar fields in nature (in spite of the fact that most attractive unified theories of nature require the existence of scalar fields in the low energy effective Lagrangian). Scalar fields were initially introduced to particle physics to yield an order parameter for the symmetry breaking phase transition. Many phase transitions exist in nature; however, in all cases, the order parameter is a condensate. Hence, it is useful to consider the possibility of obtaining inflation using condensates, and in particular to ask if this would yield a different inflationary scenario.

The analysis of a theory with condensates is intrinsically non-perturbative. The expectation value of the Hamiltonian $\langle H\rangle$ of the theory contains terms with arbitrarily high powers of the expectation value $\langle\varphi\rangle$ of the condensate. A recent study of the possibility of obtaining inflation in a theory with condensates was undertaken in [96] (see also [97] for some earlier work). Instead of truncating the expansion of $\langle H\rangle$ at some arbitrary order, the assumption was made that the expansion of $\langle H\rangle$ in powers of $\langle\varphi\rangle$ is asymptotic and, specifically, Borel summable (on general grounds one expects that the expansion will be asymptotic - see e.g. [98])

$$
\begin{aligned}
\langle H\rangle & =\sum_{n=0}^{\infty} n !(-1)^{n} a_{n}\left\langle\varphi^{n}\right\rangle \\
& =\int_{0}^{\infty} d s \frac{f(s)}{s\left(s m_{p l}+\langle\varphi\rangle\right)} e^{-1 / s} .
\end{aligned}
$$

The first line represents the original series, the second line the resummed series. The function $f(s)$ is determined by the coefficients $a_{n}$.

The cosmological scenario is as follows: the expectation value $\langle\varphi\rangle$ vanishes at times before the phase transition when the condensate forms. Afterwards, $\langle\varphi\rangle$ evolves according to the classical equations of motion with the potential given by (46) (assuming that the kinetic term assumes its standard form). It can easily be checked that the slow rolling conditions are satisfied. However, the slow roll conditions remain satisfied for all values of $\langle\varphi\rangle$, thus leading to a graceful exit problem - inflation will never terminate.

However, correlation functions, in particular $\left\langle\phi^{2}\right\rangle$, are in general infrared divergent in the de Sitter phase of an expanding Universe. One must therefore introduce a phenomenological cutoff parameter $\epsilon(t)$ into the vacuum expectation value (VEV), and replace $\langle\varphi\rangle$ by $\langle\varphi\rangle / \epsilon$. It is natural to take $\epsilon(t) \sim H(t)$ (see e.g. $[99,100])$. Hence, the dynamical system consists of two coupled functions of time $\langle\varphi\rangle$ and $\epsilon$. A careful analysis shows that a graceful exit from inflation occurs precisely if $\langle H\rangle$ tends to zero when $\langle\varphi\rangle$ tends to large values.

As is evident, the scenario for inflation in this composite field model is very different from the standard potential-driven inflationary scenario. It is particularly interesting that the graceful exit problem from inflation is linked to the cosmological constant problem. Note that models of inflation based on composites presumably do not suffer from the trans-Planckian problem, the reason being that the effective field theory which describes the strongly interacting system is timetranslation-invariant during the de Sitter phase. The physical picture is that mode interactions are essential, and are responsible for generating the fluctuations on a scale $k$ when this scale leaves the Hubble radius at time $t_{i}(k)$.

\section{VI.B Nonsingular Universe Construction}

Another possibility of obtaining inflation is by making use of a modified gravity sector. More specifically, we can add to the usual gravitational action higher derivative curvature terms. These terms become important only at high curvatures. As realized a long time ago [23], specific choices of the higher derivative terms can lead to inflation. It is well motivated to consider effective gravitational actions with higher derivative terms when studying the properties of spacetime at large curvatures, since any effective action for classical gravity obtained from string theory, quantum gravity, or by integrating out matter fields, contains such terms. In our context, the interesting question is whether one can obtain a version of inflation avoiding some of the problems discussed in the previous section, specifically whether one can obtain nonsingular cosmological models. 
Most higher derivative gravity theories have much worse singularity problems than Einstein's theory. However, it is not unreasonable to expect that in the fundamental theory of nature, be it string theory or some other theory, the curvature of space-time is limited. In Refs. [101, 102] the hypothesis was made that when the limiting curvature is reached, the geometry must approach that of a maximally symmetric space-time, namely de Sitter space. The question now becomes whether it is possible to find a class of higher derivative effective actions for gravity which have the property that at large curvatures the solutions approach de Sitter space. A nonsingular Universe construction which achieves this goal was proposed in Refs. $[103,104]$. It is based on adding to the Einstein action a particular combination of quadratic invariants of the Riemann tensor chosen such that the invariant vanishes only in de Sitter space-times. This invariant is coupled to the Einstein action via a Lagrange multiplier field in a way that the Lagrange multiplier constraint equation forces the invariant to zero at high curvatures. Thus, the metric becomes de Sitter and hence geodesically complete and explicitly nonsingular.

If successful, the above construction will have some very appealing consequences. Consider, for example, a collapsing spatially homogeneous Universe. According to Einstein's theory, this Universe will collapse in a finite proper time to a final "big crunch" singularity. In the new theory, however, the Universe will approach a de Sitter model as the curvature increases. If the Universe is closed, there will be a de Sitter bounce followed by re-expansion. Similarly, spherically symmetric vacuum solutions of the new equations of motion will presumably be nonsingular, i.e., black holes would have no singularities in their centers. In two dimensions, this construction has been successfully realized [105].

The nonsingular Universe construction of [103, 104] and its applications to dilaton cosmology [106, 107] are reviewed in a recent proceedings article [108]. What follows is just a very brief summary of the points relevant to the problems listed in Section V.

The procedure for obtaining a nonsingular Universe theory [103] is based on a Lagrange multiplier construction. Starting from the Einstein action, one can introduce Lagrange multipliers fields $\varphi_{i}$ coupled to selected curvature invariants $I_{i}$, and with potentials $V_{i}\left(\varphi_{i}\right)$ chosen such that at low curvature the theory reduces to Einstein's theory, whereas at high curvatures the solutions are manifestly nonsingular. The minimal requirements for a nonsingular theory are that all curvature invariants remain bounded and the space-time manifold is geodesically complete.

It is possible to achieve this by a two-step procedure. First, we choose a curvature invariant $I_{1}\left(g_{\mu \nu}\right)$ (e.g. $I_{1}=R$ ) and demand that it be explicitly bounded by the $\varphi_{1}$ constraint equation. In a second step, we demand that as $I_{1}\left(g_{\mu \nu}\right)$ approaches its limiting value, the metric $g_{\mu \nu}$ approach the de Sitter metric $g_{\mu \nu}^{D S}$, a definite nonsingular metric with maximal symmetry. In this case, all curvature invariants are automatically bounded (they approach their de Sitter values), and the space-time can be extended to be geodesically complete. The second step can be implemented by [103] choosing a curvature invariant $I_{2}\left(g_{\mu \nu}\right)$ with the property that

$$
I_{2}\left(g_{\mu \nu}\right)=0 \Leftrightarrow g_{\mu \nu}=g_{\mu \nu}^{D S}
$$

introducing a second Lagrange multiplier field $\varphi_{2}$ which couples to $I_{2}$, and choosing a potential $V_{2}\left(\varphi_{2}\right)$ which forces $I_{2}$ to zero at large $\left|\varphi_{2}\right|$ :

$$
S=\int d^{4} x \sqrt{-g}\left[R+\varphi_{1} I_{1}+V_{1}\left(\varphi_{1}\right)+\varphi_{2} I_{2}+V_{2}\left(\varphi_{2}\right)\right],
$$

with asymptotic conditions

$$
\begin{aligned}
V_{1}\left(\varphi_{1}\right) & \sim \varphi_{1} \text { as }\left|\varphi_{1}\right| \rightarrow \infty \\
V_{2}\left(\varphi_{2}\right) & \sim \text { const as }\left|\varphi_{2}\right| \rightarrow \infty \\
V_{i}\left(\varphi_{i}\right) & \sim \varphi_{i}^{2} \text { as }\left|\varphi_{i}\right| \rightarrow 0 \quad i=1,2 .
\end{aligned}
$$

The first constraint renders $R$ finite, the second forces $I_{2}$ to zero, and the third is required in order to obtain the correct low curvature limit.

The invariant

$$
I_{2}=\left(4 R_{\mu \nu} R^{\mu \nu}-R^{2}+C^{2}\right)^{1 / 2},
$$

singles out the de Sitter metric among all homogeneous and isotropic metrics (in which case adding $C^{2}$, the Weyl tensor square, is superfluous), all homogeneous and anisotropic metrics, and all radially symmetric metrics.

As a specific example one can consider the action [103, 104]

$$
\begin{aligned}
S= & \int d^{4} x \sqrt{-g} \\
& {\left[R+\varphi_{1} R-\left(\varphi_{2}+\frac{3}{\sqrt{2}} \varphi_{1}\right) I_{2}^{1 / 2}+V_{1}\left(\varphi_{1}\right)+V_{2}\left(\varphi_{2}\right)\right] }
\end{aligned}
$$

with

$$
\begin{aligned}
& V_{1}\left(\varphi_{1}\right)=12 H_{0}^{2} \frac{\varphi_{1}^{2}}{1+\varphi_{1}}\left(1-\frac{\ln \left(1+\varphi_{1}\right)}{1+\varphi_{1}}\right) \\
& V_{2}\left(\varphi_{2}\right)=-2 \sqrt{3} H_{0}^{2} \frac{\varphi_{2}^{2}}{1+\varphi_{2}^{2}} .
\end{aligned}
$$

It can be shown that all solutions of the equations of motion which follow from this action are nonsingular $[103,104]$. They are either periodic about Minkowski space-time $\left(\varphi_{1}, \varphi_{2}\right)=(0,0)$ or else asymptotically approach de Sitter space $\left(\left|\varphi_{2}\right| \rightarrow \infty\right)$.

One of the most interesting properties of this theory is asymptotic freedom [104], i.e., the coupling between matter and gravity goes to zero at high curvatures. It is easy to add matter (e.g., dust, radiation or a scalar 
field) to the gravitational action in the standard way. One finds that in the asymptotic de Sitter regions, the trajectories of the solutions projected onto the $\left(\varphi_{1}, \varphi_{2}\right)$ plane are unchanged by adding matter. This applies, for example, in a phase of de Sitter contraction when the matter energy density is increasing exponentially but does not affect the metric. The physical reason for asymptotic freedom is obvious: in the asymptotic regions of phase space, the space-time curvature approaches its maximal value and thus cannot be changed even by adding an arbitrarily high matter energy density. Hence, there is the possibility that this theory will admit a natural suppression mechanism for cosmological fluctuations. If this were the case, then the solution of the singularity problem would at the same time help resolve the fluctuation problem of potential-driven inflationary cosmology.

\section{VI.C Back-Reaction of Cosmological Perturba- tions}

The linear theory of cosmological perturbations in inflationary cosmology is well studied. However, effects beyond linear order have received very little attention. Beyond linear order, perturbations can effect the background in which they propagate, an effect well known from early studies [109] of gravitational waves. As will be summarized below, the back-reaction of cosmological perturbations in an exponentially expanding Universe acts like a negative cosmological constant, as first realized in the context of studies of gravitational waves in de Sitter space in [110].

Gravitational back-reaction of cosmological perturbations concerns itself with the evolution of space-times which consist of small fluctuations about a symmetric Friedmann-Robertson-Walker space-time with metric $g_{\mu \nu}^{(0)}$. The goal is to study the evolution of spatial averages of observables in the perturbed space-time. In linear theory, such averaged quantities evolve like the corresponding variables in the background space-time. However, beyond linear theory perturbations have an effect on the averaged quantities. In the case of gravitational waves, this effect is well-known [109]: gravitational waves carry energy and momentum which affects the background in which they propagate. Here, we shall focus on scalar metric perturbations.

The idea behind the analysis of gravitational backreaction [111] is to expand the Einstein equations to second order in the perturbations, to assume that the first order terms satisfy the equations of motion for linearized cosmological perturbations [33] (hence these terms cancel), to take the spatial average of the remaining terms, and to regard the resulting equations as equations for a new homogeneous metric $g_{\mu \nu}^{(0, b r)}$ which includes the effect of the perturbations to quadratic order:

$$
G_{\mu \nu}\left(g_{\alpha \beta}^{(0, b r)}\right)=8 \pi G\left[T_{\mu \nu}^{(0)}+\tau_{\mu \nu}\right]
$$

where the effective energy-momentum tensor $\tau_{\mu \nu}$ of gravitational back-reaction contains the terms resulting from spatial averaging of the second order metric and matter perturbations:

$$
\tau_{\mu \nu}=<T_{\mu \nu}^{(2)}-\frac{1}{8 \pi G} G_{\mu \nu}^{(2)}>,
$$

where pointed brackets stand for spatial averaging, and the superscripts indicate the order in perturbations.

As formulated in (57) and (58), the back-reaction problem is not independent of the coordinatization of space-time and hence is not well defined. It is possible to take a homogeneous and isotropic space-time, choose different coordinates, and obtain a non-vanishing $\tau_{\mu \nu}$. This "gauge" problem is related to the fact that in the above prescription, the hypersurface over which the average is taken depends on the choice of coordinates.

The key to resolving the gauge problem is to realize that to second order in perturbations, the background variables change. A gauge independent form of the back-reaction equation (57) can hence be derived [111] by defining background and perturbation variables $Q=Q^{(0)}+\delta Q$ which do not change under linear coordinate transformations. Here, $Q$ represents collectively both metric and matter variables. The gauge-invariant form of the back-reaction equation then looks formally identical to (57), except that all variables are replaced by the corresponding gauge-invariant ones. We will follow the notation of [33], and use as gauge-invariant perturbation variables the Bardeen potentials [35] $\Phi$ and $\Psi$ which in longitudinal gauge coincide with the actual metric perturbations $\delta g_{\mu \nu}$. Calculations hence simplify greatly if we work directly in longitudinal gauge. These calculations have been confirmed [112] by working in a completely different gauge, making use of the covariant approach.

In [113], the effective energy-momentum tensor $\tau_{\mu \nu}$ of gravitational back-reaction was evaluated for long wavelength fluctuations in an inflationary Universe in which the matter responsible for inflation is a scalar field $\varphi$ with the potential

$$
V(\varphi)=\frac{1}{2} m^{2} \varphi^{2}
$$

Since in this model there is no anisotropic stress, the perturbed metric in longitudinal gauge can be written [33] in terms of a single gravitational potential $\phi$

$$
d s^{2}=(1+2 \phi) d t^{2}-a(t)^{2}(1-2 \phi) \delta_{i j} d x^{i} d x^{j},
$$

where $a(t)$ is the cosmological scale factor.

It is now straightforward to compute $G_{\mu \nu}^{(2)}$ and $T_{\mu \nu}^{(2)}$ in terms of the background fields and the metric and matter fluctuations $\phi$ and $\delta \varphi$, By taking averages and making use of (58), the effective energy-momentum tensor $\tau_{\mu \nu}$ can be computed [113]. 
The general expressions for the effective energy density $\rho^{(2)}=\tau_{0}^{0}$ and effective pressure $p^{(2)}=-\frac{1}{3} \tau_{i}^{i}$ involve many terms. However, they greatly simplify if we consider perturbations with wavelength greater than the Hubble radius. In this case, all terms involving spatial gradients are negligible. From the theory of linear cosmological perturbations (see e.g. [33]) it follows that on scales larger than the Hubble radius the time derivative of $\phi$ is also negligible as long as the equation of state of the background does not change. The Einstein constraint equations relate the two perturbation variables $\phi$ and $\delta \varphi$, enabling scalar metric and matter fluctuations to be described in terms of a single gauge-invariant potential $\phi$. During the slow-rolling period of the inflationary Universe, the constraint equation takes on a very simple form and implies that $\phi$ and $\delta \varphi$ are proportional. The upshot of these considerations is that $\tau_{\mu \nu}$ is proportional to the two point function $\left\langle\phi^{2}\right\rangle$, with a coefficient tensor which depends on the background dynamics. In the slow-rolling approximation we obtain [113]

$$
\rho^{(2)} \simeq-4 V<\phi^{2}>
$$

and

$$
p^{(2)}=-\rho^{(2)} .
$$

This demonstrates that the effective energy-momentum tensor of long-wavelength cosmological perturbations has the same form as a negative cosmological constant.

Note that during inflation, the phase space of infrared modes is growing. Hence, the magnitude of $\left|\rho^{(2)}\right|$ is also increasing. Hence, the back-reaction mechanism may lead to a dynamical relaxation mechanism for a bare cosmological constant driving inflation [114]. A similar effect holds for pure gravity at two loop order in the presence of a bare cosmological constant [110].

The interpretation of $\rho^{(2)}$ as a local density has been criticized, e.g. in [115]. Instead of computing physical observables from a spatially averaged metric, one should compute the spatial average of physical invariants corrected to quadratic order in perturbation theory. Work on this issue is in progress [116] (see also [68]).

\section{Conclusions}

Inflationary cosmology is an attractive scenario. It solves some problems of standard cosmology and leads to the possibility of a causal theory of structure formation. The specific predictions of an inflationary model of structure formation, however, depend on the specific realization of inflation, which makes the idea of inflation hard to verify or falsify. Many models of inflation have been suggested, but at the present time none are sufficiently distinguished to form a "standard" inflationary theory.

There is now a consistent quantum theory of the generation and evolution of linear cosmological pertur- bations which describes the origin of fluctuations from an initial vacuum state of the fluctuation modes at the beginning of inflation, and which forms the basis for the precision calculations of the power spectrum of density fluctuations and of CMB anisotropies which allow detailed comparisons with current and upcoming observations.

As explained in Section IV, a new theory of inflationary reheating (preheating) has been developed based on parametric resonance. Preheating leads to a rapid energy transfer between the inflaton field and matter at the end of inflation, with important cosmological consequences. Recent developments in this area are the realization that long wavelength gravitational fluctuations may be amplified exponentially in models with an entropy perturbation mode which is not suppressed during inflation, and the study of the effects of noise in the inflaton field on the resonance process, leading to the result that such noise actually enhances the resonance (this result also leads to a new proof of Anderson localization).

Note, however, that there are important conceptual problems for scalar field-driven inflationary models. Four such problems discussed in Section V are the fluctuation problem, the trans-Planckian problem, the singularity problem and the cosmological constant problem, the last of which is the Achilles heel of these inflationary models.

It may be that a convincing realization of inflation will have to wait for an improvement in our understanding of fundamental physics. Some promising but incomplete avenues which address some of the problems mentioned above and which yield inflation are discussed in Section VI.

\section{Acknowledgements}

I wish to thank Prof. J.A.S. Lima for the invitation to present this lecture, and for his wonderful hospitality during my visit to Brazil. I also wish to thank Prof. Richard MacKenzie for hospitality at the Université de Montreal and Profs. Jim Cline and Rob Myers for hospitality at McGill University during the time this manuscript was completed. The author is supported in part by the US Department of Energy under Contract DE-FG02-91ER40688, Task A.

\section{References}

[1] A. Guth, Phys. Rev. D 23, 347 (1981).

[2] A. Linde, 'Particle Physics and Inflationary Cosmology' (Harwood, Chur, 1990).

[3] S. Blau and A. Guth, 'Inflationary Cosmology,' in '300 Years of Gravitation' ed. by S. Hawking and W. Israel (Cambridge Univ. Press, Cambridge, 1987).

[4] K. Olive, Phys. Rep. 190, 307 (1990). 
[5] A. Liddle and D. Lyth, 'Cosmological Inflation and Large-Scale Structure' (Cambridge Univ. Press, Cambridge, 2000).

[6] D. Lyth and A. Riotto, Phys. Rept. 314, 1 (1999).

[7] R. Brandenberger, 'Inflationary Cosmology: Progress and Problems', hep-ph/9910410.

[8] R. Brandenberger, 'Status Review of Inflationary Cosmology', hep-ph/0101119.

[9] D. Kazanas, Ap. J. 241, L59 (1980).

[10] W. Press, Phys. Scr. 21, 702 (1980).

[11] G. Chibisov and V. Mukhanov, 'Galaxy Formation and Phonons,' Lebedev Physical Institute Preprint No. 162 (1980);

G. Chibisov and V. Mukhanov, Mon. Not. R. Astron. Soc. 200, 535 (1982).

[12] V. Lukash, Pis'ma Zh. Eksp. Teor. Fiz. 31, 631 (1980).

[13] K. Sato, Mon. Not. R. Astron. Soc. 195, 467 (1981).

[14] R. Brandenberger, Phys. Lett. B 129, 397 (1983).

[15] A. Linde, Phys. Lett. B 108, 389 (1982).

[16] A. Albrecht and P. Steinhardt, Phys. Rev. Lett. 48, 1220 (1982).

[17] A. Linde, Phys. Lett. B 129, 177 (1983).

[18] A. Linde, Phys. Rev. D 49, 748 (1994).

[19] P. Binetruy and G. Dvali, Phys. Lett. D 388, 241 (1996);

E. Halyo, Phys. Lett. B 387, 43 (1996);

A. Linde and A. Riotto, Phys. Rev. D 56, R1844 (1997).

[20] T. Banks, 'Remarks on M Theoretic Cosmology', hepth/9906126.

[21] P. Horava and E. Witten, Nucl. Phys. B 460, 506 (1996).

[22] G. Dvali and S. Tye, Phys. Lett. B 450, 72 (1999); N. Kaloper and A. Linde, Phys. Rev. D 59, 101303 (1999).

[23] A. Starobinski, Phys. Lett. B 91, 99 (1980).

[24] M. Gasperini and G. Veneziano, Astropart. Phys. 1, 317 (1993).

[25] T. Damour and V. Mukhanov, Phys. Rev. Lett. 80, 3440 (1998).

[26] V. Mukhanov and G. Chibisov, JETP Lett. 33, 532 (1981).

[27] A. Guth and S.-Y. Pi, Phys. Rev. Lett. 49, 110 (1982); S. Hawking, Phys. Lett. B 115, 295 (1982);

A. Starobinski, Phys. Lett. B 117, 175 (1982);

V. Mukhanov, JETP Lett. 41, 493 (1985).

[28] J. Bardeen, P. Steinhardt and M. Turner, Phys. Rev. D 28, 1809 (1983).

[29] R. Sachs and A. Wolfe, Ap. J. 147, 73 (1967).

[30] E. Harrison, Phys. Rev. D 1, 2726 (1970); Ya.B. Zel'dovich, Mon. Not. R. astron. Soc. 160, 1p (1972). 396, L1
[31] E. Lifshitz, Zh. Eksp. Teor. Fiz. 16, 587 (1946);

E. Lifshitz and I. Khalatnikov, Adv. Phys. 12, 185 (1963).

[32] W. Press and E. Vishniac, Ap. J. 239, 1 (1980).

[33] V. Mukhanov, H. Feldman and R. Brandenberger, Phys. Rep. 215, 203 (1992).

[34] R. Brandenberger, H. Feldman, V. Mukhanov and T. Prokopec, 'Gauge Invariant Cosmological Perturbations: Theory and Applications,' publ. in "The Origin of Structure in the Universe," eds. E. Gunzig and P. Nardone (Kluwer, Dordrecht, 1993).

[35] J. Bardeen, Phys. Rev. D 22, 1882 (1980).

[36] R. Brandenberger, R. Kahn and W. Press, Phys. Rev. D 28, 1809 (1983).

[37] H. Kodama and M. Sasaki, Prog. Theor. Phys. Suppl. No. 78, 1 (1984).

[38] R. Durrer and N. Straumann, Helvet. Phys. Acta 61, 1027 (1988);

R. Durrer, Fund. Cosmic Phys. 15, 209 (1994), astroph/9311040.

[39] D. Lyth, Phys. Rev. D 31, 1792 (1985);

D. Lyth and M. Mukherjee, Phys. Rev. D 38, 485 (1988).

[40] J. Hwang and E. Vishniac, Ap. J. 353, 1 (1990).

[41] G.F.R. Ellis and M. Bruni, Phys. Rev. D 40, 1804 (1989);

G.F.R. Ellis, J. Hwang and M. Bruni, Phys. Rev. D 40, 1819 (1989).

[42] D. Salopek and J. Stewart, Phys. Rev. D 51, 517 (1995).

[43] J. Stewart, Class. Quantum Grav. 7, 1169 (1990).

[44] J. Stewart and M. Walker, Proc. R. Soc. A 341, 49 (1974).

[45] R. Brandenberger and R. Kahn, Phys. Rev. D 28, 2172 (1984).

[46] R. Brandenberger, "Modern Cosmology and Structure Formation", in "CP Violation and the Limits of the Standard Model (TASI 94)', ed. J. Donoghue (World Scientific, Singapore, 1995), astro-ph/9411049; R. Brandenberger, in 'Physics of the Early Universe,' proc. of the 1989 Scottish Univ. Summer School in Physics, ed. by J. Peacock, A. Heavens and A. Davies (SUSSP Publ., Edinburgh, 1990);

R. Brandenberger, 'Lectures on Modern Cosmology and Structure Formation', in 'Particles and Fields', ed. by O. Eboli and V. Ribelles (World Scientific, Singapore 1994).

[47] J. Martin and D. Schwarz, Phys. Rev. D 57, 3302 (1998);

M. Gotz, Mon. Not. Roy. Astron. Soc. 295, 873 (1998), astro-ph/9704271.

[48] F. Finelli and R. Brandenberger, Phys. Rev. Lett. 82, 1362 (1999).

[49] J. Bardeen, unpublished (1984).

[50] R. Brandenberger, Nucl. Phys. B 245, 328 (1984).

[51] M. Sasaki, Prog. Theor. Phys. 76, 1036 (1986).

[52] V. Mukhanov, Zh. Eksp. Teor. Fiz. 94, 1 (1988). 
[53] F. Adams, K. Freese and A. Guth, Phys. Rev. D 43, 965 (1991).

[54] A. Dolgov and A. Linde, Phys. Lett. B 116, 329 (1982).

[55] L. Abbott, E. Farhi and M. Wise, Phys. Lett. B 117, 29 (1982).

[56] J. Traschen and R. Brandenberger, Phys. Rev. D 42, 2491 (1990).

[57] L. Landau and E. Lifshitz, 'Mechanics' (Pergamon, Oxford, 1960);

V. Arnold, 'Mathematical Methods of Classical Mechanics' (Springer, New York, 1978).

[58] L. Kofman, A. Linde and A. Starobinski, Phys. Rev. Lett. 73, 3195 (1994).

[59] Y. Shtanov, J. Traschen and R. Brandenberger, Phys. Rev.D 51, 5438 (1995).

[60] L. Kofman, A. Linde and A. Starobinski, Phys. Rev.D 56, 3258 (1997).

[61] P. Greene, L. Kofman, A. Linde and A. Starobinski, Phys. Rev. D 56, 6175 (1997).

[62] S. Khlebnikov and I. Tkachev, Phys. Rev. Lett. 77, 219 (1996);

S. Khlebnikov and I. Tkachev, Phys. Lett. B 390, 80 (1997);

T. Prokopec and T. Roos, Phys. Rev. D 55, 3768 (1997);

B. Greene, T. Prokopec and T. Roos, Phys. Rev.D 56, 6484 (1997).

[63] G. Felder and L. Kofman, 'The Development of Equilibrium after Preheating', hep-ph/0011160.

[64] B. Bassett, D. Kaiser and R. Maartens, Phys. Lett. B 455, 84 (1999).

[65] M. Parry and R. Easther, Phys. Rev. D 59, 061301 (1999).

[66] Y. Nambu and A. Taruya, Prog. Theor. Phys. 97, 83 (1997).

[67] H. Kodama and Hamazaki, Prog. Theor. Phys. 96, 1123 (1996).

[68] N. Afshordi and R. Brandenberger, 'Super- Hubble Nonlinear Perturbations During Inflation', grqc/0011075.

[69] W. Lin, X. Meng and X. Zhang, Phys. Rev. D 61, 121301 (2000), hep-ph/9912510.

[70] K. Jedamzik and G. Sigl, Phys. Rev.D 61, 023519 (2000), hep-ph/9906287.

[71] P. Ivanov, Phys. Rev. D61, 023505 (2000), astro$\mathrm{ph} / 9906415$.

[72] A. Liddle, D. Lyth, K. Malik and D. Wands, Phys. Rev.D 59, 123501 (1999), hep-ph/9912473.

[73] B. Bassett and F. Viniegra, Phys. Rev. D62, 043507 (2000), hep-ph/9909353.

[74] F. Finelli and R. Brandenberger, Phys. Rev. D62, 083502 (2000), hep-ph/0003172;

D. Wands, K. Malik, D. Lyth and A. Liddle, Phys. Rev. D 62, 043527 (2000), astro-ph/0003278.
[75] J. Zibin, R. Brandenberger and D. Scott, 'Back Reaction and the Parametric Resonance of Cosmological Fluctuations', Phys. Rev. D (in press) (2001), hepph/0007219.

[76] V. Zanchin, A. Maia, W. Craig and R. Brandenberger, Phys. Rev. D57, 4651 (1998).

[77] V. Zanchin, A. Maia, W. Craig and R. Brandenberger, Phys. Rev. D60, 023505 (1999).

[78] W. Craig and R. Brandenberger, in preparation (2001).

[79] P. Anderson, Phys. Rev. 109, 1 (1958).

[80] T. Jacobson, 'Black Hole Evaporation: An Open Question', in Brighton 1990, Relativistic Astrophysics, Cosmology, and Fundamental Physics, Proc. of the 1990 TEXAS/ESO-CERN symposium, ed. by J. Barrow, L. Mestel and P. Thomas (New York Acad. Sci., New York, 1991).

[81] W. Unruh, Phys. Rev. D51, 2827 (1995).

[82] S. Corley and T. Jacobson, Phys. Rev. D 54, 1568 (1996);

S. Corley, Phys. Rev.D 57, 6280 (1998).

[83] R. Brandenberger and J. Martin, 'The Robustness of Inflation to Changes in Super-Planck-Scale Physics', astro-ph/0005432.

[84] J. Martin and R. Brandenberger, 'The Transplanckian Problem of Inflationary Cosmology', hep-th/0005209.

[85] J. Martin and R. Brandenberger, 'A Cosmological Window on Trans-Planckian Physics', astro-ph/0012301.

[86] A. Borde and A. Vilenkin, Phys. Rev. Lett. 72, 3305 (1993).

[87] S. Weinberg, Rev. Mod. Phys. 61, 1 (1989); S. Carroll, W. Press and E. Turner, Ann. Rev. Astron. Astrophys. 30, 499 (1992);

S. Carroll, 'The Cosmological Constant', astro$\mathrm{ph} / 0004075$.

[88] J. Moffatt, Int. J. Mod. Phys. D2, 351 (1995).

[89] A. Albrecht and J. Magueijo, Phys. Rev. D59, 0433516 (1999).

[90] R. Brustein and G. Veneziano, Phys. Lett. B329, 429 (1994);

R. Easther, K. Maeda and D. Wands, Phys. Rev. D53, 4247 (1996);

N. Kaloper, R. Madden and K. Olive, Phys. Lett. B371, 34 (1995).

[91] N. Kaloper, A. Linde and R. Bousso, Phys. Rev. D59, 043508 (1999).

[92] A. Buonanno, T. Damour and G. Veneziano, Nucl. Phys. B543, 275 (1999).

[93] E. Kiritsis, JHEP 9910:010 (1999), hep-th/9906206.

[94] A. Kehagias and E. Kiritsis, JHEP 9911:022 (1999), hep-th/9910174.

[95] S. Alexander, JHEP 0011:017 (2000), hepth/9912037.

[96] R. Brandenberger and A. Zhitnitsky, Phys. Rev. D55, 4640 (1997). 
[97] R. Ball and A. Matheson, Phys. Rev. D45, 2647 (1992);

A. Matheson, R. Ball, A.-C. Davis and R. Brandenberger, Nucl. Phys.B 328, 223 (1989).

[98] A. Zhitnitsky, Phys. Rev. D54, 5148 (1996).

[99] A. Linde, Phys. Lett. B116, 335 (1982).

[100] A. Vilenkin and L. Ford, Phys. Rev.D 25, 2569 (1982).

[101] M. Markov, Pis'ma Zh. Eksp. Theor. Fiz. 36, 214 (1982);

M. Markov, Pis'ma Zh. Eksp. Theor. Fiz. 46, 342 (1987);

V. Ginsburg, V. Mukhanov and V. Frolov, Pis'ma Zh. Eksp. Theor. Fiz. 94, 3 (1988);

V. Frolov, M. Markov and V. Mukhanov, Phys. Rev. D41, 383 (1990).

[102] D. Morgan, Phys. Rev. D45, R1005 (1992).

[103] V. Mukhanov and R. Brandenberger, Phys. Rev. Lett. 68, 1969 (1992).

[104] R. Brandenberger, V. Mukhanov and A. Sornborger, Phys. Rev. D 48, 1629 (1993).

[105] M. Trodden, V. Mukhanov and R. Brandenberger, Phys. Lett.B 316, 483 (1993).

[106] R. Brandenberger, R. Easther and J. Maia, JHEP 9808, 7 (1998), gr-qc/9806111.
[107] D. Easson and R. Brandenberger, JHEP 9909:003 (1999), hep-th/9905175.

[108] R. Brandenberger and J. Magueijo, 'Imaginative cosmology', hep-ph/9912247.

[109] D. Brill and J. Hartle, Phys. Rev. 135 B271-278 (1964); R. Isaacson, Phys. Rev. 166, 1263 and 1272 (1968).

[110] N. Tsamis and R. Woodard, Phys. Lett. B292, 269 (1993); Phys. Rev. D54, 2621 (1996); Nucl. Phys.B 474, 235 (1996); Ann. Phys. 253, 1 (1997).

[111] V. Mukhanov, L. R. W. Abramo and R. Brandenberger, Phys. Rev. Lett. 78, 1624 (1997).

[112] L.R.W. Abramo and R. Woodard, Phys. Rev.D 60, 044010 (1999), astro-ph/9811430.

[113] L. R. W. Abramo, R. Brandenberger and V. Mukhanov, Phys. Rev. D56, 3248 (1997), grqc/9704037.

[114] R. Brandenberger, 'Back Reaction of Cosmological Perturbations', hep-th/0004016.

[115] W. Unruh, 'Cosmological Long Wavelength Perturbations', astro-ph/9802323.

[116] L.R.W. Abramo and R. Woodard, in preparation (2001). 